\title{
La organización de la asistencia sanitaria a pacientes crónicos con pluripatología: La experiencia de Ourense
}

\author{
Organization of the medical care of chronic patients with plurypatology. \\ Ourense experience
}

\author{
Manuel de Toro Santos, Salomé Gil Rodríguez, Consuelo Benito Torres, Pilar Rozas Lozano \\ Servicio de Medicina Interna. Complexo Hospitalario Universitario de Ourense (CHOU). SERGAS. Ourense
}

\section{RESUMEN}

El envejecimiento poblacional y la mayor expectativa de vida desencadenan un cambio en las necesidades asistenciales de todos los Sistemas de Salud. En la provincia de Ourense la tasa de envejecimiento es la más alta de la autonomía gallega alcanzando un $29.5 \%$ de personas mayores de 65 años, lo estimado para Europa en el año 2050. Ante esa realidad desde el Servicio de Medicina Interna del Complexo Hospitalario Universitario de Ourense se impulsan cambios en la asistencia sanitaria a la población, proponiendo un modelo mixto que aglutina la experiencia de las unidades clínicas de atención médica integral desarrolladas en Andalucía y la forma de trabajo de las unidades de pluripatología.

PALABRAS CLAVE: Medicina Interna, continuidad asistencial, internista de referencia, Atención Primaria.

\section{Introducción}

La filosofía de la medicina interna se centra en la visión del enfermo en su conjunto y no de la enfermedad en particular, así como en promover la continuidad asistencial, fundamentalmente en pacientes con patologías múltiples, crónicas 0 de avanzada edad, y la coordinación y el liderazgo de equipos multidisciplinares como queda bien definido en el programa de formación de nuestra especialidad y en las recomendaciones del plan estratégico de nuestra sociedad de medicina interna 1,2. Los internistas debemos tener la capacidad de adaptación y de innovación para reorientar nuestra forma de trabajo y saber atender las necesidades sanitarias de la población. Los cambios demográficos actuales, con el envejecimiento poblacional y las mayores expectativa de vida, protagonizan un cambio en las necesidades asistenciales de todos los Sistemas de Salud en la atención a los pacientes con edad avanzada y con pluripatología que nos obligan a modernizar y reorientar nuestros servicios de medicina para una nueva forma de trabajo integrado en la Comunidad y claramente orientado hacia las necesidades de las personas, aportando los valores de nuestro perfil profesional como internistas ${ }^{3}$.

\section{Nuestra realidad}

Nuestro Servicio se encuentra en plena fase de transformación al constituirse en un Servicio único procedente de dos servicios de medicina que trabajaban con formas y objetivos diferentes. En la actualidad integrado en el Complexo Hospitalario Universitario de Ourense, de segundo nivel con más de 800 camas, nos encontramos en la situación especial de

\begin{abstract}
The aging process of the population and the increase of life expectancy triggered a change in the health care needs of all Health Systems. The aging rate of the region of Ourense is the highest one among the regions in Galicia. It reaches $29.5 \%$ of people over 65 years which is the predicted percentage for Europe in 2050. Having this evidence into account, the Internal Medicine Service of the University Hospital Complex of Ourense promotes changes in health care population, suggesting a mixed model that brings together the experience of clinical units for total medical treatment developed in Andalucía, and the way of working in comorbidity units. KEY WORDS: Internal Medicine, care continuity, referring doctor of internal medicine, Primary Care.
\end{abstract}

orientar nuestra misión como la de un servicio de medicina interna para el área sanitaria de Ourense. Partimos de una situación asistencial fragmentada con lugares diversos de hospitalización y consultas, distribuidos en el Hospital Cristal, Hospital Santa María Nai y en el Hospital Materno Infantil. En las Unidades de Hospitalización del Hospital Cristal, en donde está ubicado el servicio de urgencias y servicios de UCl y reanimación y servicios quirúrgicos, ingresan de forma preferente los pacientes con patologías que por sus características de complejidad o inestabilidad hemodinámica puedan requerir apoyo de dichas unidades, además de los ingresos convencionales como en las demás zonas de hospitalización. La población de referencia para nuestro servicio es de 239.000 habitantes y muestra la tasa de envejecimiento más alta de nuestra Autonomía, con datos de tarjeta sanitaria a fecha 1 de enero de 2012 que muestran un $29.5 \%$ de personas mayores de 65 años (Tabla 1), reflejando una realidad que es la estimada para Europa en el 2050. Tiene un especial interés conocer la edad media de los pacientes que atendemos en nuestro servicio como queda reflejado en el porcentaje de altas en el año 2011 y que se puede comparar con el resto se servicios de medicina interna de los hospitales de la comunidad autónoma (Tabla2), comprobando que es en el grupo de las personas mayores de 65 años donde se generan el 88\% de los ingresos hospitalarios en nuestro Servicio. El tercer punto de relieve es saber que hacemos en nuestra labor asistencial del paciente hospitalizado a través de nuestra lista de GRDs (Tabla 3), que nos enseñan que los diez más frecuen- 
Tabla 1: Distribución poblacional por edad en Galicia y Provincias

\begin{tabular}{|c|c|c|c|c|c|c|c|c|}
\hline & & \multicolumn{2}{|c|}{$\leq 14$} & \multicolumn{2}{|c|}{$15-64$} & \multicolumn{2}{|c|}{$\geq 65$} & \multirow{2}{*}{ Total } \\
\hline & & $n$ & Porcentaje (\%) & $n$ & Porcentaje (\%) & $n$ & Porcentaje (\%) & \\
\hline \multirow[t]{3}{*}{ A CORUÑA } & Hombre & 68.261 & 12,42 & 377.156 & 68,61 & 104.291 & 18,97 & 549.708 \\
\hline & Mujer & 64.297 & 10,83 & 383.628 & 64,59 & 145.983 & 24,58 & 593.908 \\
\hline & Total & 132.558 & 11,59 & 760.784 & 66,52 & 250.274 & 21,88 & 1.143 .616 \\
\hline \multirow[t]{3}{*}{ LUGO } & Hombre & 16.569 & 9,99 & 107.822 & 65,01 & 41.474 & 25,00 & 165.865 \\
\hline & Mujer & 15.863 & 9,02 & 104.731 & 59,55 & 55.284 & 31,43 & 175.878 \\
\hline & Total & 32.432 & 9,49 & 212.553 & 62,20 & 96.758 & 28,31 & 341.743 \\
\hline \multirow[t]{3}{*}{ OURENSE } & Hombre & 16.000 & 10,10 & 100.623 & 63,52 & 41.779 & 26,38 & 158.402 \\
\hline & Mujer & 15.299 & 8,91 & 100.793 & 58,70 & 55.608 & 32,39 & 171.700 \\
\hline & Total & 31.299 & 9,48 & 201.416 & 61,02 & 97.387 & 29,50 & 330.102 \\
\hline \multirow[t]{3}{*}{ PONTEVEDRA } & Hombre & 63.838 & 13,71 & 324.300 & 69,63 & 77.593 & 16,66 & 465.731 \\
\hline & Mujer & 59.878 & 12,09 & 324.190 & 65,46 & 111.145 & 22,44 & 495.213 \\
\hline & Total & 123.716 & 12,87 & 648.490 & 67,48 & 188.738 & 19,64 & 960.944 \\
\hline \multirow[t]{3}{*}{ GALICIA } & Hombre & 164.668 & 12,29 & 909.901 & 67,92 & 265.137 & 19,79 & 1.339 .706 \\
\hline & Mujer & 155.337 & 10,81 & 913.342 & 63,57 & 368.020 & 25,62 & 1.436 .699 \\
\hline & Total & 320.005 & 11,53 & 1.823 .243 & 65,67 & 633.157 & 22,80 & 2.776 .405 \\
\hline
\end{tabular}

Fuente: Servicio Galego de Saúde. Población con tarjeta sanitaria a 01/01/2012

tes suponen el $45 \%$ de nuestra actividad asistencial. Con la información de la que disponemos no hay duda alguna que nuestros principales pacientes se caracterizan por una edad mayor de 65 años y con patologías crónicas que precisan de ingreso por descompensación de las mismas, siendo los GRDs de Insuficiencia Respiratoria $(541+540+88+101)$ y los de Insuficiencia cardiaca $(127+544+87)$ los que representan el mayor porcentaje de altas, dato ya referenciado en el estudio de hospitales del sistema de salud español4. Tomando como punto de corte los pacientes que habían ingresado más de 3 veces en el área médica en nuestro hospital, hemos visto que 380 pacientes, el 6.9\% del total, habían generado el 20.6\% de todas las estancias en el área médica. Es de prever, con la experiencia recogida de las diversas unidades de pluripatología, que una actuación organizada y coordinada sobre los pacientes frágiles con pluripatología ayudaría a evitar un alto número de ingresos y disminuir las estancias hospitalarias ${ }^{5}$. Con estos datos y si tuviéramos que definir lo que hacemos los internistas, tendríamos que decir que empleamos la mayoría de nuestro tiempo asistencial en cuidar a pacientes de edad avanzada y con enfermedades crónicas y por lo tanto hemos de adaptar nuestra práctica diaria al mejor cuidado de los mismos y a una mejor gestión de los recursos sanitarios en el área de salud. Sin duda es para nosotros una oportunidad que no debemos desaprovechar.

Al realizar el proyecto del servicio de medicina interna para el complexo hospitalario de Ourense y el área sanitaria, además de contar con los cambios demográficos y epidemiológicos y mayor prevalencia de las enfermedades crónicas ${ }^{6}$, hay que contemplar otras variables como los cambios sociales y las expectativas de los ciudadanos que demandan más y mejores servicios, los nuevos criterios de formas de trabajo en la organización, buscando la implicación de todos los profesionales en la gestión y el uso de las nuevas tecnologías ${ }^{7,8}$. El escenario económico actual, con restricciones presupuestarias y recursos limitados, nos obliga a una utilización adecuada de los recursos para la asistencia. Éste aspecto va a condicionar nuestro proyecto de una forma muy importante y tendremos que echar mano de la imaginación y búsqueda de nuevas propuestas para lograr la mayor eficiencia del sistema además de la implicación imprescindible de la administración. Los cambios en la práctica clínica estableciendo el principio de autonomía del paciente y el principio de equidad nos llevan a buscar la eficiencia. Por otra parte la evolución en los patrones de asistencia sanitaria marcan cambios sustanciales con la aparición de alternativas a la hospitalización como los hospitales y unidades de día, hospitalización a domicilio (HADO), telemedicina, tele-asistencia, cirugía ambulatoria, unidades de estancias cortas, consultas de acto único ${ }^{9}$. Las innovaciones en tecnología de la comunicación, las bases de datos y el desarrollo informático, con la implantación de historia electrónica, dan paso, además de lo estrictamente asistencial, a facilitar la investigación clínica y el desarrollo de nuevas posibilidades de gestión de recursos humanos y económicos y sobre todo a anular la barrera entre niveles asistenciales que genera un sinfín de disfunciones en detrimento de la eficiencia. 
Tabla 2: Distribución de las altas por edad en los servicios de medicina interna de los hospitales de Galicia Datos codificados de enero a noviembre de 2011

\begin{tabular}{|l|c|c|c|c|c|c|c|c|c|}
\hline \multirow{2}{*}{} & \multicolumn{2}{|c|}{$15-44$ años } & \multicolumn{2}{c|}{$45-64$ años } & \multicolumn{2}{c|}{$65-74$ años } & \multicolumn{2}{c|}{$>74$ años } & \multirow{2}{*}{ Total no altas } \\
\cline { 2 - 9 } & $N^{0}$ altas & $\%$ altas & $N^{0}$ altas & $\%$ altas & No altas & $\%$ altas & No altas & $\%$ altas & \\
\hline A CORUÑA & 575 & $6,32 \%$ & 1.601 & $17,60 \%$ & 1.799 & $19,78 \%$ & 5.120 & $56,29 \%$ & 9.095 \\
\hline OURENSE & 218 & $3,78 \%$ & 461 & $7,99 \%$ & 686 & $11,90 \%$ & 4.402 & $76,33 \%$ & 5.767 \\
\hline SANTIAGO & 303 & $7,02 \%$ & 674 & $15,61 \%$ & 645 & $14,94 \%$ & 2.696 & $62,44 \%$ & 4.318 \\
\hline MARCIDE & 279 & $6,60 \%$ & 853 & $20,19 \%$ & 906 & $21,44 \%$ & 2.187 & $51,76 \%$ & 4.225 \\
\hline PONTEVEDRA & 191 & $5,22 \%$ & 387 & $10,57 \%$ & 457 & $12,48 \%$ & 2.626 & $71,73 \%$ & 3.661 \\
\hline VIGO & 251 & $8,42 \%$ & 542 & $18,18 \%$ & 552 & $18,52 \%$ & 1.636 & $54,88 \%$ & 2.981 \\
\hline CALDE & 136 & $4,93 \%$ & 362 & $13,13 \%$ & 400 & $14,51 \%$ & 1.857 & $67,38 \%$ & 2.756 \\
\hline SALNES & 116 & $6,88 \%$ & 276 & $16,36 \%$ & 276 & $16,36 \%$ & 1.019 & $60,40 \%$ & 1.687 \\
\hline BARBANZA & 86 & $5,24 \%$ & 228 & $13,90 \%$ & 357 & $21,77 \%$ & 969 & $59,09 \%$ & 1.640 \\
\hline VERIN & 30 & $2,79 \%$ & 114 & $10,61 \%$ & 201 & $18,72 \%$ & 729 & $67,88 \%$ & 1.074 \\
\hline XUNQUEIRA & 39 & $3,63 \%$ & 134 & $12,49 \%$ & 203 & $18,92 \%$ & 696 & $64,86 \%$ & 1.073 \\
\hline Hospital da Costa & 60 & $5,67 \%$ & 188 & $17,75 \%$ & 178 & $16,81 \%$ & 633 & $59,77 \%$ & 1.059 \\
\hline VALDEORRAS & 31 & $2,94 \%$ & 135 & $12,80 \%$ & 153 & $14,50 \%$ & 736 & $69,76 \%$ & 1.055 \\
\hline MONFORTE & 12 & $1,62 \%$ & 60 & $8,11 \%$ & 93 & $12,57 \%$ & 575 & $77,70 \%$ & 740 \\
\hline Total general & 2.327 & $5,66 \%$ & 6.015 & $14,62 \%$ & 6.906 & $16,79 \%$ & 25.881 & $62,92 \%$ & 41.131 \\
\hline
\end{tabular}

Fuente: Grupo Conjunto Mínimo Básico de Datos (CMBD) de hospitalización de agudos

\section{La necesidad y la oportunidad de los cambios}

Hoy en día la máxima aportación para un mejor funcionamiento del Sistema de Salud, en concreto en el ámbito asistencial, sería conseguir una completa coordinación entre el hospital y atención primaria. Siempre se habla de que esta relación tiene que mejorar y también de las dificultades para lograrlo; la última iniciativa está representada por el Documento de la Declaración de Sevilla que pretende contribuir a sensibilizar a la población, a los profesionales y a las administraciones sanitarias a facilitar e impulsar y desarrollar sistemas de atención sanitaria basados en la atención integral y continuidad asistencial contando con una población informada y responsable ${ }^{10}$.

La hospitalización convencional "esperando a los pacientes que ingresan a través del servicio de urgencias" debe dar paso a una forma de trabajo diferente en la que hospitalización puede ser parte de la necesidad asistencial pero no la única forma, ya que existen alternativas a la hospitalización con experiencias clínicas que evidencian un mejor aprovechamiento de los recursos sociosanitarios y mostrando que los problemas asistenciales también pueden resolverse en la consulta externas de alta resolución, unidades de día, hospitales de día, por consulta telefónica, correo electrónico 0 a través de reuniones con los médicos de atención primaria en los centros de salud de referencia 9 . Esta forma de trabajo ya se ha implantado en hospitales de España desde los años 90 y han puesto de relieve que se puede trabajar de una forma integrada y con buenos resultados de gestión clínica. La implantación de las Unidades Clínicas de Atención Médica Integral (UCAMI) en Andalucía ya tienen una experiencia de más de 15 años. Los promotores de esta iniciativa cuentan cómo se generó:" Entre los médicos de atención primaria y del hospital un nuevo estilo de trabajo en equipo en el que el concepto de facultativo especialista de área adquiría una nueva dimensión en base a la sectorización de la población y la toma de conciencia de que el especialista no es solo el referente de un equipo médico sino el responsable de la atención prestada en su área de conocimiento en la población a su cargo" ${ }^{\text {"5 } 11,12 .}$

Se acordó la existencia de un internista de referencia accesible telefónicamente, personalización de todas las consultas o derivaciones, posibilidad de ingreso directo desde el centro o el domicilio, posibilidad de consultas clínicas puntuales telefónicas, demora diagnóstica acordada con posibilidad de valoración urgente durante la mañana, sesiones clínicas conjuntas quincenales y participación en la toma de decisiones importantes referidas al paciente. La evolución posterior fue hacia la creación de La Unidad Clínica de Atención Médica Integral. Esta forma de trabajo hace que el paciente sea el centro del sistema sanitario y en todo momento no pierda la perspectiva de quien es su médico en el centro de salud y en el hospital. Profesionales y pacientes han valorado positivamente esta forma de trabajar en las comunidades en las que se ha implantado y creemos que la posibilidad de poner en marcha en nuestra Provincia este modelo de trabajo, aprovechando el momento de reorganizar nuestro servicio, puede ser la oportunidad para iniciar los cambios tan deseados de lograr la coordinación de atención primaria y el hospital en la provincia de Ourense.

\section{Nuestra forma de llevarlo a cabo}

Tras ser conocedores de la realidad asistencial de la que somos responsables y con la clara decisión de que hay que llevar a cabo los cambios organizativos para lograr una Asistencia Integrada en el área sanitaria que atendemos, hemos Ilevado 
Tabla 3: Descripción, frecuencias y estancias de los 10 GRDs más frecuentes en el servicio de Medicina Interna del Complexo hospitalario de Ourense

\begin{tabular}{|c|l|c|c|c|c|c|}
\hline GRD & Descripción de los 10 GRD & Peso GRD & Altas & $\begin{array}{c}\% \text { sobre total } \\
\text { altas }\end{array}$ & $\begin{array}{c}\text { Estancias } \\
\text { 541 }\end{array}$ & $\begin{array}{c}\text { Estancia } \\
\text { media }\end{array}$ \\
\hline 127 & Insunquía simple y otros trastornos respiratorios exceptuando & 2,3431 & 883 & $14,12 \%$ & 9.711 & 11,00 \\
\hline 544 & Insuficiencia cardiaca y shock & 1,4222 & 360 & $5,76 \%$ & 3.110 & 8,64 \\
\hline 89 & Neumonía simple y pleuritis en edad mayor de 17 años con CC & 1,3134 & 228 & $3,65 \%$ & 2.197 & 9,64 \\
\hline 540 & $\begin{array}{l}\text { Infecciones e inflamaciones respiratorias excepto neumonía } \\
\text { simple con CC mayor }\end{array}$ & 3,3679 & 217 & $3,47 \%$ & 2.553 & 11,76 \\
\hline 87 & Edema pulmonar e insuficiencia respiratoria & 1,5093 & 183 & $2,93 \%$ & 1.755 & 9,59 \\
\hline 14 & Ictus con infarto & 1,8799 & 178 & $2,85 \%$ & 1.767 & 9,93 \\
\hline 101 & Otros diagnósticos de aparato respiratorio con CC & 0,9993 & 173 & $2,77 \%$ & 1.436 & 8,30 \\
\hline 533 & $\begin{array}{l}\text { Otros trastornos del sistema nervioso excepto AIT, convulsiones } \\
\text { y cefalea con CC mayor }\end{array}$ & 4,4301 & 165 & $2,64 \%$ & 2.290 & 13,88 \\
\hline 88 & Enfermedad pulmonar obstructiva crónica & 1,1469 & 162 & $2,59 \%$ & 1.478 & 9,12 \\
\hline & Total de los 10 GRD & 2,2105 & 2.816 & $45,03 \%$ & 29.185 & 10,36 \\
\hline & Total MEDICINA INTERNA & 1,949 & 6.254 & 100,00 & 65.117 & 10,41 \\
\hline
\end{tabular}

Fuente: Grupo Conjunto Mínimo Básico de Datos (CMBD) de hospitalización de agudos

a cabo reuniones con los servicios de atención primaria para conocer sus necesidades y explorar la posibilidad avanzar en las nuevas formas de colaboración asistencial. Históricamente contamos con una buena interrelación de una gran mayoría de médicos de atención primaria con los que de forma habitual trabajamos con reuniones en los centros de salud, posibilidad de consultas telefónicas, interconsultas programadas de común acuerdo y que ven también la necesidad de una mejor relación asistencial, aportando el profesionalismo y teniendo al paciente como referente fundamental del proceso asistencial. Somos conocedores de que no solo se busca la relación entre médicos, se va más allá, es la relación entre todos los profesionales que trabajan en red con el paciente y familia: enfermería, asistencia social, fisioterapeutas. Éste modelo andaluz, por así llamarlo, nos parece muy adecuado y coherente ya que se basa fundamentalmente en establecer una relación entre profesionales con criterios de responsabilidad, calidad, seguridad para el paciente y establece un camino que es válido para la atención de cualquier proceso asistencial y no solo el de pacientes pluripatológicos. Trabajar en equipo hoy más que nunca es innovación ${ }^{13}$.

Hemos afrontado reuniones periódicas entre los 32 internistas que conformamos nuestro Servicio para debatir el cambio de modelo organizativo y asistencial y afrontar las líneas estratégicas esenciales que debemos tener en cuenta para el futuro desarrollo de nuestra especialidad en el hospital y la comunidad, básicamente:

- La colaboración estrecha y la convergencia de objetivos con atención primaria.

- La asistencia integral y con continuidad al grupo de pacientes vulnerables, pluripatológicos y de edad avanzada.
- El refuerzo de la presencia del internista como consultor en el hospital.

Se llevaron a cabo diversas reuniones con la Dirección del Centro y posteriormente, tras la implantación de la Gerencia del Área Integrada de Hospitales y Atención Primaria, con la nueva Dirección de Área a quién se ha explicado el nuevo modelo asistencial, que encaja perfectamente en la política integradora de niveles asistenciales, siendo asumidos desde la misma los cambios fundamentales que nos parecen primordiales para acometer este nuevo modelo de continuidad asistencial diseñado en las UCAMI'1

- Sectorizar la población con un internista de referencia para cada centro de salud.

- Creación de la Unidad de día que aglutina las consultas de alta resolución y Hospital de día.

- Reorganización del trabajo asistencial de los internistas para simultanear diariamente la atención de pacientes en camas de hospitalización y en consultas o en Hospital de Día.

Por nuestra parte tenemos por delante gran cantidad de tareas que realizar, propias de éste modelo que acabo de reseñar, algunas ya en marcha y otras en fase de elaboración, con el fin de interrelacionar nuestro trabajo dentro y fuera del hospital a través de acuerdos de colaboración con Ios demás servicios y unidades, como urgencias y HADO y lograr el desarrollo de trabajo multidisciplinar que permita que pacientes que ingresan en éstas unidades con descompensaciones de enfermedades crónicas como bronquitis crónica e insuficiencia cardiaca sean reconducidos hacia una continuidad asistencial con su internista de referencia, hacia la consulta y el hospital de día y su propio médico de 
Familia. El trabajar en equipo con enfermería es esencial e imprescindible cuando afrontamos el proceso asistencial del paciente con pluripatología. La elaboración de los planes de cuidados estandarizados y personalizados, el informe de continuidad asistencial, los roles de enfermera referente, enfermera colaboradora y enfermera de enlace así como la gestión compartida en equipos multidisciplinarios son pasos obligados a conseguir en este proyecto. Ya contamos con una herramienta básica que es la informatización de la historia clínica electrónica compartida (IANUS), que da acceso del médico de familia a todo el sistema de información clínica del hospital, pero todavía nos debe dar la posibilidad de tener un sistema de identificación de pacientes pluripatológicos, sistemas de alerta ante ingresos y altas hospitalarias de los pacientes pluripatológicos dirigidas tanto al internista, como a atención primaria y a la enfermera gestora de casos. Desde la propia historia del paciente debe facilitarse la posibilidad de interconsultas del médico de familia al internista de referencia con la posibilidad de resolver los problemas sin que el paciente esté presente. La conexión telefónica a través de teléfono móvil corporativo para la programación de ingresos y consultas puntuales no demorables por parte de los compañeros de Atención Primaria es una aproximación más a la colaboración en el cuidado de los pacientes.

Para llevar a cabo este proyecto se ha diseñado una sectorización de la población, mayor de 14 años, en cinco sectores asistenciales de acuerdo a la frecuentación de consultas, hospitalización y edad poblacional y contemplando que cada sector tenga una asistencia sanitaria en una zona de salud de la capital y la otra en una comarca, con un número entre 40.000 y 50.000 habitantes. Cada sector de población tendrá como referencia para la atención sanitaria en el hospital a una sección de medicina interna constituida por 4 internistas y personal de enfermería que estarán relacionados con los profesionales de los correspondientes centros de salud y con el objetivo de brindar continuidad asistencial a sus pacientes, con las formas de trabajo mencionadas y que cuentan con espacios de trabajo para hospitalización, consultas externas y hospital de día.

\section{Unidad de pacientes pluripatologicos}

Puede parecer paradójico plantear la creación de una unidad de pluripatología tras establecer anteriormente como forma de trabajo el modelo de una unidad clínica de atención médica integrada, en donde el proceso de atención al paciente pluripatológico sería un proceso integrado más. Hemos dudado mucho en ésta decisión pero en este momento necesitamos un motor impulsor en ésta área de conocimiento que es el de cuidar más que curar. Las personas mayores a las que atendemos precisan que les aportemos nuestros mejores conocimientos, lo más adecuado para cada caso y cuando no podemos más paliar sus dolencias. La especial tasa de envejecimiento de nuestra población con la consiguiente prevalencia de la pluripatología, nos lleva a la necesidad de crear la cultura de la atención al paciente pluripatológico a través de la creación de una unidad de pluripatología (UPP) que se encargue de adaptar en nuestro medio las experiencias que ya han dado sus frutos en otras comunidades y buscando la interrelación con todos los profesionales del ámbito socio sanitario, desarrollando ideas para conseguir el mejor grado de asistencia a nuestros mayores entre todos. En España los servicios de salud de las comunidades autónomas han introducido iniciativas para dar respuesta asistencial a este reto con ejemplos como la UCAMI o la Unidad de Pacientes con Pluripatología y Atención Médica Integral (UPPAMI) ${ }^{5,11}$, que pueden valer como modelos a seguir adaptándolas a nuestra realidad socio sanitaria. A nosotros nos parece adecuado establecer una fórmula mixta en la que de forma habitual el método de trabajo es el de una unidad clínica de atención integral y a la que se suma una unidad de pacientes con pluripatología.

El Ministerio de acuerdo con el trabajo de un grupo de expertos que han dedicado tiempo y experiencia profesional a desarrollar formulas nuevas de atención a los pacientes pluripatológicos ha publicado los estándares y recomendaciones de la UPP, atendiendo a un concepto organizativo y definiéndola "como una organización de profesionales sanitarios, que ofrece atención multidisciplinaria mediante un amplio espectro de modalidades de asistencia a pacientes frágiles con pluripatología, y que cumple unos requisitos funcionales, estructurales y organizativos, que garantizan las condiciones adecuadas de calidad, seguridad y eficiencia, para realizar esta actividad"14.

Uno de los objetivos de ésta unidad será la de reorientar nuestra actividad en el campo asistencial y del conocimiento de las enfermedades crónicas con la determinación de implicar a todos los estamentos relacionados en la misma. Nuestra idea viene reforzada por documento de Consenso de la las sociedades de Medicina Interna y Medicina Familiar y Comunitaria ${ }^{11}$ que apuesta por un modelo de integración total para lograr el cambio y en el que deben estar representadas las especialidades sanitarias implicadas en el proceso de atención a pacientes crónicos que tendrán que adaptar su perfil competencial a las nuevas necesidades. Algunos profesionales como geriatras llevan mucho tiempo haciendo esta función y debemos ser capaces de aprender de su experiencia y trabajar juntos. Los farmacólogos clínicos, nutricionistas, fisioterapeutas, psicólogos y trabajadores sociales tienen que hacer notar su protagonismo asistencial así como todos los profesionales implicados en la rehabilitación y la terapia ocupacional en pacientes crónicos por la necesidad de evitar la dependencia en pacientes con enfermedad crónica. El 
resto de objetivos de ésta unidad van enfocados a aspectos asistenciales y de recuperación funcional de los pacientes con la visión de un trabajo en equipo, integrador de todos los profesionales antes mencionados.

Para ello contamos con una unidad de hospitalización constituida por 3 médicos internistas y una geriatra y una Unidad de enfermería y se dispone actualmente de 32 camas de hospitalización de las que 12 serán para utilización de recuperación funcional, siendo la interrelación con el equipo Interconsultor del área quirúrgica esencial para derivación a la misma de los pacientes en el momento más adecuado ${ }^{15,16,17}$.

El resto de la hospitalización atendería de forma preferente o específicamente a los ingresos de pacientes institucionalizados en centros socio-sanitarios, que ingresan por descompensación de su patología crónica, facilitando la relación con los mismos. Si en el marco asistencial la coordinación entre atención primaria y hospital es escasa, entre los centros sociosanitarios y el hospital es prácticamente inexistente. Queremos desde esta unidad coordinarnos de una forma activa con las residencias geriátricas para establecer un marco de colaboración con el objetivo de brindar una continuidad asistencial a los pacientes. Existen experiencias de trabajo con residencias geriátricas donde un médico y enfermera con formación en geriatría dan apoyo en la valoración geriátrica integral y visitas de seguimiento a los pacientes ancianos más complejos de forma programada y a demanda, revisión y adecuación del tratamiento farmacológico conjuntamente con el médico de la residencia y desarrollo de protocolos consensuados para situaciones clínicas frecuentes (patología respiratoria crónica, infección urinaria, tratamientos paliativos) además de reuniones periódicas de coordinación con el personal médico y de enfermería de las residencias geriátricas y gestión de casos conjuntamente con especialistas, que han dado muy buenos resultados disminuyendo la frecuentación de urgencias en un $49 \%$ y la hospitalización urgente en un $48 \%$ así como el gasto de farmacia en un $9 \% 18$.

La posibilidad de iniciar una experiencia de trabajo en conjunto con el servicio de psiquiatría está muy avanzado tanto en la zona de hospitalización como en la consulta externa, con la posibilidad de atender a los pacientes con cuadros confusionales en la unidad con visita diaria del psiquiatra e internista y estableciendo protocolo de actuación diagnóstica y tratamiento así como de continuidad asistencial19.

La consulta externa está orientada a pacientes con edad avanzada y pluripatología con la finalidad de hacer siempre una valoración geriátrica integral y desde ahí recomendar el plan de actuación destinado a evitar el deterioro funcional. Los pacientes pueden ser derivados desde atención primaria, de los demás servicios y secciones de medicina interna del hospital y de los centros sociosanitarios y una especial rela- ción con el servicio de urgencias y HADO para seguimiento de pacientes identificados como pluripatológicos que han presentado descompensación de sus enfermedades crónicas y que precisan continuidad asistencial.

Se dispone de una local para la consulta, hospital de día y consulta de enfermería y proximidad con la consulta de asistencia social. El trabajo de enfermería es muy importante tanto en la propia zona de consulta, donde se encargará de la recepción de los pacientes que van a ser atendidos toma las constantes y valoración integral de su estado funcional, cognitivo, valoración, nutricional y cumplimiento de la medicación, como en el acto de relación con las enfermeras de los centros de salud correspondiente y con la enfermera gestora de casos para hacer el seguimiento de los pacientes en conjunto.

\section{Internista consultor en el hospital}

Al mismo tiempo que nuestro servicio de medicina interna se organiza para dar solución a las primera línea estratégica de darle un sentido de continuidad asistencial con atención primaria y por lo tanto un sentido de trabajo en la comunidad, acometemos otros cambios como el de potenciar el papel de internista consultor en el hospital buscando no solo resolver un aspecto asistencial y remarcar la importancia de nuestro trabajo como internistas sino como agente cohesionador de los servicios hospitalarios y atención primaria.

Tras una época en que la interconsulta sobre los problemas de un paciente se establecía de forma oral entre el médico responsable de la atención del paciente y el médico consultor directamente 0 a través de una sesión clínica, si la complejidad del caso lo requería, se ha pasado a una forma general de relación de petición de interconsulta por escrito 0 electrónica, sin que a veces hablen entre si los médicos implicados en la asistencia del paciente ${ }^{20}$. Ésta labor asistencial tiene ciertas características como la de no estar programada, tener un carácter discontinuo y no registrado en la actividad hospitalaria y dificultades como la falta de comunicación entre profesionales, los diferentes criterios de valorar la pertinencia de la interconsulta urgente 0 el establecimiento de quién es la responsabilidad directa del paciente o manejo conjunto del mismo etc ${ }^{21}$. De destacar positivamente la comprobación de la disminución de mortalidad y morbilidad y acortamiento de las estancia media recomendándose la incorporación de internistas a los servicios quirúrgicos. ${ }^{22,23}$

En nuestro hospital se había iniciado la figura del internista consultor en el área quirúrgica hace años y en este momento de cambio en nuestra organización vemos esta función como una oportunidad no solo para potenciar nuestro papel de consultor sino como figura integradora y de cohesión de la asistencia en el hospital. Observamos un aumento continuo de interconsultas a medicina interna por los servicios quirúrgicos 
de nuestro hospital, que no se explica por el aumento de ingresos en dichos servicios, y que parece estar en relación con el incremento de la edad y la comorbilidad de los enfermos. Un gran porcentaje de los pacientes con pluripatología que atendemos en nuestras plantas de hospitalización y consultas son los mismos que ocupan camas quirúrgicas con fracturas 0 intervenciones programadas y que presentan luego las complicaciones posquirúrgicas como neumonías, confusión, sépsis, tromboembolismos etc. Estamos en la tarea de rediseñar el manejo de estos pacientes, de una forma proactiva, formando un equipo integrado por médicos, enfermera y asistente social, que permita su valoración integral permitiendo ver cuáles son sus necesidades y medidas que debemos poner en marcha, para recobrar el estado funcional que tenía previo al ingreso, ya que de ello se deriva la mayor autonomía y mejor pronóstico22. Desde está unidad se debe llevar a cabo la derivación de los pacientes, de una forma protocolizada y consensuada, para garantizar la continuidad asistencial con su médico y enfermero del centro de atención primaria 0 si lo requiere en las demás secciones de medicina interna, unidad de recuperación funcional de la unidad de pluripatología, HADO. En el momento actual la unidad de interconsulta quirúrgica está formada por dos médicos y trabajadora de asistencia social a demanda y necesitamos completarla con una enfermera que se encargue de la valoración integral y sirva de enlace con los demás estamentos asistenciales del hospital y Atención Primaria.

La implantación de este proyecto debe estar finalizado en la primavera de 2012 y comenzar a ver sus frutos en el próximo año.

Finalmente como internistas no quisiéramos dejar de recordar que para que todo esto tenga algo de sentido es preciso mantener nuestro profesionalismo y humanismo en iguales dosis. Nuestro trabajo como médicos consiste en ser los valedores de personas que depositan su confianza en nosotros, en el campo de la salud, y es preciso que antes de nada sepamos hacer bien las "pequeñas cosas" con el paciente; cosas tan sencillas como presentarnos, darle la mano, sentarnos y escucharle, interesarnos por él, romper barreras mostrando humildad, en suma ser médicos. ${ }^{24.25}$

\section{Bibliografía}

1. Ministerio de Sanidad y política Social. Programa Formativo de la especialidad de Medicina Interna. Orden SCO/227/2007. Boe. n 33,7 de febrero de 2007

2. García Alegría J, Conthe Gutiérrez P. Orientación estratégica de la Sociedad Española de Medicina Interna. Rev Clin Esp. 2011; 211: 46-51.

3. Arcadi Gual, Albert Oriol-Bosch, Helios Pardell. El Médico del Futuro. Med Clin Barc 2010; 134: 363-368.

4. Barba Martina R, Marco Martínez J, Emilio Losa J, Canora Lebrato J, Plaza Canteli S y Zapatero Gaviria A. Análisis de 2 años de actividad de Medicina Interna en los hospitales del Sistema Nacional de Salud. Rev Clin Esp. 2009; 209: 459-466.

5. Medina Asensio J .Experiencia de la UPP del Hospital 12 de Octubre. Gestión y Evaluación de Costes Sanitarios. Vol.11, Monográfico 2, Junio 2010.

6. García-Morillo JS, Bernabeu-Wittel M, Ollero-Baturone M, Aguilar-Guisad M, RamírezDuque N, González de la Puente MA et al. Incidencia y características clínicas de los pacientes con pluripatología ingresados en una unidad de medicina interna. Med Clin (Barc). 2005; 125: 5-9

7. Castillo Rueda A, Portugal Álvarez J. Proyecto técnico de gestión y funcionamiento de la unidad asistencial de Medicina Interna. An Med Interna.2004. 21: 31-38.

8. Bernabeu-Wittel M, García Morillo S, Ollero M, Hernández-Quero J, González de la Puente MA, Montero Pérez-Barquero M, et al. El Plan Estratégico para el desarrollo de la Medicina Interna en Andalucía. Rev Clin Esp. 2008; 208: 295-301.

9. Torres Salinas M, Capdevila Morel JA, Armario García P, Montull Morer S et al. Alternativas a la hospitalización convencional en medicina interna. Med Clin (Barc). 2005 124: 620-6.

10. Ollero Baturone M, Orozco Beltrán D. Domingo Rico C, Román Sánchez P, López Soto A, Melguizo Jiménez M, et al. Declaración de Sevilla. Conferencia nacional para la atención al paciente con enfermedades crónicas. Rev Clin Esp. 2011; 211: 604-6.

11. Fernández Moyano A, García Garmendia JL, Palmero Palmero C, García Vargas-Machuca B, Páez Pinto JM, Álvarez Alcina M, et al. Continuidad asistencial. Evaluación de un programa de colaboración entre Atención Hospitalaria y Atención Primaria. Rev Clin Esp. 2007; 207: 510-20.

12. Ollero Baturone M, Álvarez Tello M, Barón Franco B; Bernabéu Wittel M; Codina Lanaspa A, Fernández Moyano A, Garrido Porras E, Ortiz Camúñez MA, Rojas García de Paso J, Romero Alonso A, Romero Carmona S, Sanz Amores R Atención a pacientes pluripatológicos: proceso asistencial integrado $2^{\mathrm{a}}$ ed.: Consejería de Salud de Andalucía, 2007. ISBN: 978-84-690-6500-6.

13. Working Together-That's Innovation! Howard K. Rabinowitz, MD. Ann Intern Med. 2004; 140: $660-661$.

14. Ministerio de Sanidad y Política Social. Unidad de Pacientes Pluripatológicos. Estándares y recomendaciones. Madrid: Ministerio de Sanidad y Política Social; 2009. www. msc.es/organizacion/sns/planCalidadSNS/docs/EyR_UPP.

15. González Montalvo J, Alarcón Alarcón T, Pallardo Rodil B, Gotor Pérez P, Mauleón Álvarez de Linera JL, Gil Garay E. Ortogeriatría en pacientes agudos (I). Aspectos asistenciales. Rev Esp Geriatr Gerontol. 2008; 43: 239-51.

16. González Montalvo Jl, Alarcón Alarcón T, Pallardo Rodil B, Gotor Pérez P, Pareja Sierra T. Ortogeriatría en pacientes agudos (II). Aspectos clínicos. Rev Esp Geriatr Gerontol. 2008 Sep-Oct; 43: 316-29.

17. Gotor Pérez P. Abordaje multidisciplinar de la fractura de cadera. La importancia de la enfermera en los nuevos modelos asistenciales. Rev Esp Geriatr Gerontol. 2011; 46: 287-288.

18. Díaz-Gegúndeza M, Paluzie G, Sanz-Ballester C, Boada-Mejorana M, Terré-Ohmee $S$ Ruiz-Pozae D. Evaluación de un programa de intervención en residencias geriátricas para reducir la frecuentación hospitalaria. Rev Esp Geriatr Gerontol. 2011; 46: 261-264.

19. Martínez Calvo A, Carrera S, Stein B, Huyse F, Herzog T, Lobo A et al. El proyecto multinacional europeo y multicéntrico español de mejora de calidad asistencial en psiquiatría de enlace en el hospital general: el perfil clínico en España. Med Clin (Barc) 2000; 115: 690-4.

20. Navia-Osorio García-Braga JM. Acerca de la interconsulta medica. Med Clin (Barc) 2012; 138: 133-137.

21. Montero Ruiz E, López-Álvarez J. La interconsulta médica: problemas y soluciones. Med Clin (Barc). 2011; 136: 488-490.

22. Montero Ruiz E, Hernández Ahijado C, López Álvarez J. Efecto de la adscripción de internistas a un servicio quirúrgico. Med Clin (Barc). 2005; 124: 332-5.

23. Monte Secades R, Rabuñal Rey R, Rigueiro Veloso MT, García País MJ, Casariego Vales E, Guerrero Lombardı J. Papel del internista como consultor de servicios Quirúrgicos. Rev Clin Esp. 2004; 204: 345-50.

24. Larry R. Churchill, and David Schenck. Healing Skills for Medical Practice. Ann Intern Med. 2008; 149: 720-724

25. W. Kahn M. Etiquette-Based Medicine. N Engl J Med.2008; 358; 19 